\title{
Increased expression of long non-coding RNA XIST predicts favorable prognosis of cervical squamous cell carcinoma subsequent to definitive chemoradiation therapy
}

\author{
REIKO KOBAYASHI ${ }^{1}$, RYU MIYAGAWA ${ }^{2}$, HIDEOMI YAMASHITA ${ }^{1}$, TEPPEI MORIKAWA ${ }^{2}$, \\ KAE OKUMA $^{1}$, MASASHI FUKAYAMA ${ }^{2}$, KUNI OHTOMO ${ }^{1}$ and KEIICHI NAKAGAWA ${ }^{1}$ \\ Departments of ${ }^{1}$ Radiology and ${ }^{2}$ Pathology, Graduate School of Medicine, University of Tokyo, Tokyo 113-8655, Japan
}

Received January 23, 2015; Accepted November 19, 2015

DOI: $10.3892 / \mathrm{ol} .2016 .5054$

\begin{abstract}
The present retrospective study aimed to examine the association between the expression of long non-protein-coding RNAs (lncRNAs) and clinical prognosis in the pretreatment formalin-fixed, paraffin-embedded (FFPE) tissue samples of cervical squamous cell carcinoma patients that underwent platinum-based chemoradiation therapy. Between 2001 and 2013, 49 consecutive patients with squamous cell cervical carcinoma were selected for the present study (median follow-up period, 44.1 months). The patients possessed an International Federation of Gynecology and Obstetrics stage of IB1/IIA1 (with pelvic lymph node metastasis), IB2 or IIA2-IVA, and had been treated with definitive chemoradiation therapy. The pretreatment FFPE tumor biopsies of the patients obtained diagnosis were used for analysis. Total RNAs were extracted from the FFPE tumor tissues and reverse transcription-quantitative polymerase chain reaction was performed to examine the expression level of lncRNAs. The expression level of $\mathrm{X}$ inactive-specific transcript (XIST) demonstrated a significant association with the overall survival rate $(\mathrm{P}=0.014)$. The 4 -year overall survival rates were 87.1 and $54.4 \%$ in the high and low XIST expression groups, respectively. Since the expression of XIST is associated with the overall survival rate, this IncRNA has the potential to become a predictor for the prognosis of cervical squamous cell carcinoma patients that are treated with chemoradiation therapy. Additional studies are required to investigate the underlying mechanisms of XIST that are associated with prognosis.
\end{abstract}

\section{Introduction}

Chemoradiation therapy (CRT) has become widely accepted as the standard treatment for locally advanced cervical cancer, as declared in the Clinical Announcement by the US National

Correspondence to: Dr Ryu Miyagawa, Department of Pathology, Graduate School of Medicine, University of Tokyo, 7-3-1 Hongo, Tokyo 113-8655, Japan

E-mail: ryumiya76@12.alumni.u-tokyo.ac.jp

Key words: cervical cancer, long non-coding RNA, XIST
Cancer Institute in 1999 (1,2). However, the benefits of CRT decrease with increasing International Federation of Gynecology and Obstetrics (FIGO) stage (3). Additionally, CRT may only benefit 1 out of 10 patients with cervical cancer, due to differences between tumors and the high frequency of side effects (4). Therefore, a more precise stratification of the patients that respond to CRT and the identification of prognostic markers for cervical cancer are required.

Several pretreatment variables have been associated with the clinical outcome of patients with cervical carcinoma, including the FIGO stage, tumor size, lymph node status, hemoglobin level, histological type and patient age (5). Previously, in addition to the clinicopathological factors, numerous molecular markers in cervical cancer tissue specimens or serum samples have been investigated to identify the association between the survival rate and response to CRT $(6,7)$. According to the studies reviewed by Noordhuis et $\mathrm{al}$, angiogenesis and hypoxia markers, including hypoxia-inducible factor- $1 \alpha$ and vascular endothelial growth factor, epidermal growth factor receptor pathway markers and cyclooxygenase- 2 were identified as prognostic markers, mainly by immunohistochemistry (6). In addition to protein biomarkers, which are derived from messenger RNAs (mRNAs), recent prognostic studies have investigated a large group of non-coding RNAs (ncRNAs).

Although ncRNAs are transcribed from the genomic region, they are not translated into proteins (8). In higher organisms the non-protein-coding regions of DNA are increased; in humans, $298 \%$ of DNA consists of non-protein coding regions (9), and numerous ncRNAs may be transcribed from these regions. Furthermore, a recent study has suggested that numerous types of ncRNAs are also transcribed from protein-coding regions of DNA (10). Therefore, ncRNAs are hypothesized to be important in determining the complexity of higher eukaryotes and other physiological phenomena and are considered to be as critical as mRNA coding proteins. Although the functions of ncRNAs are not well known, ncRNAs may be crucial in the development, physiology and pathology of cells, including cancer. MicroRNAs, which belong to a small group of ncRNAs, are the most well-studied ncRNAs, including microRNA-200a and microRNA-9, which have been demonstrated to be predictors of cervical cancer (11). Long non-coding RNAs 
(lncRNAs) are tentatively defined as ncRNAs >200 nucleotides in length (12-14). Numerous lncRNAs are downregulated in various cancers, which demonstrate oncogenic and tumor suppressive roles (13). There are $>601$ ncRNAs reported to be associated with cancer (13); however, the function of the majority of lncRNAs, and the association between lncRNAs and the prognosis of cervical cancer, remains to be determined (12-14).

In general, clinical tissue samples are preserved in formalin-fixed, paraffin-embedded (FFPE) blocks. Previous studies demonstrate the feasibility of quantifying gene expression by using RNA isolated from blocks of FFPE tumor tissue (15-18).

The aim of the present retrospective study was to examine expression of lncRNAs in pretreatment FFPE tissue samples of patients with cervical squamous cell carcinoma (CSCC) that underwent platinum-based CRT, and to analyze the association between lncRNA expression and the clinical prognosis of the patients.

\section{Materials and methods}

Patients and tissue samples. Between March 2001 and March 2013, consecutive patients that were treated with definitive CRT for cervical cancer at the University of Tokyo Hospital (Tokyo, Japan) and met the following eligibility criteria were selected for the present study: A diagnosis of pathologically confirmed CSCC; FFPE tumor tissue biopsies were available prior to treatment; CSCC tumors were FIGO clinical stage IB1/IIA1, with pelvic lymph node (PLN) metastasis, or stage IB2 or IIA2-IVA; the patients had undergone complete definitive CRT without any prior treatment for CSCC; and the patients had been followed-up at the University of Tokyo Hospital. Patients with distant metastasis, including para-aortic lymph node metastasis or uncontrollable other malignancies, were excluded from the present study.

Sections from the FFPE tumor blocks were stained with hematoxylin and eosin (Merck Millipore, Darmstadt, Germany), and reviewed by two experienced pathologists at the University of Tokyo Hospital to determine the suitability for analysis of the tumor content. Among the 58 patients that met the eligibility criteria, 9 were excluded from the present study, generally due to low tumor content in the FFPE samples. Therefore, 49 patients were analyzed in the current study.

The median age of the patients was 55 years (range, 29-82 years). The majority of patients were diagnosed with a FIGO stage of IIIB $(n=24)$. The median maximum tumor diameter was $5.5 \mathrm{~cm}$ (range, 2.0-9.7 cm), as measured by $\mathrm{T} 2$ weighted magnetic resonance imaging (MRI). Patient and tumor characteristics are reported in Table I.

The last patient follow-up took place in March 2014. At the time of data analysis, 34 patients $(69 \%)$ were alive and 15 patients $(31 \%)$ had succumbed to cause-specific (13 patients; $27 \%$ ) and other (2 patients; $4 \%$ ) diseases. The overall median follow-up period was 44.1 months (range, 5.2-142.1 months).

The present study was approved by the Institutional Review Board of the University of Tokyo Hospital \{approved on May 21, 2013 [no. 10152]; minor revision approved on September 9, 2013
Table I. Patient and tumor characteristics

\begin{tabular}{lr}
\hline Characteristic & $\mathrm{n}(\%)$ \\
\hline Total & $49(100)$ \\
Age range, years (median) & $29-82(55)$ \\
FIGO stage & \\
IB & $2(4)$ \\
IIA & $1(2)$ \\
IIB & $10(20)$ \\
IIIA & $7(14)$ \\
IIIB & $24(49)$ \\
IVA & $5(10)$ \\
Pelvic lymph node metastasis & $17(35)$ \\
Positive & $32(65)$ \\
Negative & \\
Initial hemoglobin range, g/dl (median) & $6.9-14.2(12.0)$ \\
Maximum tumor diameter & $2.0-9.7(5.5)$ \\
range, cm (median) & \\
Concurrent chemotherapy & \\
Tri-weekly CDDP 75 mg/m ${ }^{2}$ & $18(37)$ \\
Tri-weekly NDP $75-100 ~ m g / m^{2}$ & $18(37)$ \\
Weekly CDDP 40 mg/m² & $12(24)$ \\
Other & $35-89(45)$ \\
RT duration range, days (median) & \\
\hline
\end{tabular}

FIGO, International Federation of Gynecology and Obstetrics; CDDP, cisplatin; NDP, nedaplatin; RT, radiation therapy.

[no. 10152-(1)] and January 30, 2014 [no. 10152-(2)]\}. Written informed consent was obtained from all patients. All patient identifiers were removed prior to the analysis of the data.

$R N A$ extraction and reverse transcription-quantitative polymerase chain reaction $(R T-q P C R)$. From each FFPE block, 10-15 tissue cores, measuring $10 \mu \mathrm{m}$ in thickness, were removed. Total RNA was extracted from the tissue cores with RecoverAll ${ }^{\mathrm{TM}}$ Total Nucleic Acid Isolation kit for FFPE (Thermo Fisher Scientific, Inc., Waltham, MA, USA), according to the manufacturer's protocol. Complementary DNA (cDNA) was obtained by reverse transcribing the total RNA with a PrimeScript ${ }^{\mathrm{TM}}$ RT reagent kit with gDNA Eraser (Perfect Real Time) (Takara Bio Inc., Otsu, Shiga, Japan). RT-qPCR was performed with SYBR ${ }^{\circledR}$ Premix Ex Taq ${ }^{\mathrm{TM}}$ II (Tli RNaseH Plus; Takara Bio Inc.) and the Eco Real-Time PCR System (Illumina, Inc., San Diego, CA, USA). IncRNA values were normalized to those of GAPDH, which was used as an internal control.

lncRNAs examined. The present study focused on lncRNAs that had been implicated in various types of cancer, according to previous studies (12-14). Thus, the follow 5 lncRNAs were examined: $X$ inactive-specific transcript (XIST), Tsix, telomerase RNA component (TERC), dihydrofolate reductase (DHFR) upstream transcripts, antisense insulin-like growth-factor type-II receptor RNA (Air). 
Table II. Primer sequences for GAPDH and 5 lncRNAs.

\begin{tabular}{|c|c|}
\hline Oligonucleotides & Sequence \\
\hline \multicolumn{2}{|l|}{ GAPDH (20) } \\
\hline Foward & GCACCGTCAAGGCTGAGAAC \\
\hline Reverse & TGGTGAAGACGCCAGTGGA \\
\hline \multicolumn{2}{|l|}{ XIST (21) } \\
\hline Foward & AATGGAACGGGCTGAGTTTTAG \\
\hline Reverse & TCATCCGCTTGCGTTCATAG \\
\hline \multicolumn{2}{|l|}{ Tsix (21) } \\
\hline Foward & AGTTGTGACCGATTTGGAGGGCTTACG \\
\hline Reverse & GTATGGAGTCACCAGGTTCCCAGAGAAAGAC \\
\hline \multicolumn{2}{|l|}{ TERC $^{\mathrm{a}}$} \\
\hline Foward & TTCAGGCCGCAGGAAGAGGA \\
\hline Reverse & ACGTCCCACAGCTCAGGGAA \\
\hline \multicolumn{2}{|c|}{ DHFR upstream transcripts (19) } \\
\hline Foward & ACCTGGTCGGCTGCACCT \\
\hline Reverse & TTGCCCTGCCATGTCTCG \\
\hline \multicolumn{2}{|l|}{ Air (22) } \\
\hline Foward & GCAGCAAGAAGCACAGCAC \\
\hline Reverse & GATGTCTGCGTGGTAACTGG \\
\hline
\end{tabular}

Primers. The primer sequences used to amplify GAPDH and the 5 lncRNAs are reported in Table II. Since FFPE treatment and storage of tissue samples may lead to damage across the length of RNA, primers were used to generate amplicons that were as short as possible. These primers were selected from previous studies (19-22) or produced using GENETYX $^{\circledR}$-MAC software, version 13 (GENETYX Co., Tokyo, Japan).

Pretreatment evaluation. The pretreatment state of the patients was clinically evaluated by physical and pelvic examination without anesthesia, biopsy of the primary tumor, complete blood cell count and biochemistry profile, computed tomography (CT) of the chest, abdomen and pelvis, MRI of the pelvis, drip infusion pyelography, cystoscopy and rectoscopy. [18F]-fluoro-2-deoxy-D-glucose positron emission tomography (FDG-PET) was routinely performed from 2008. PLNs $>10 \mathrm{~mm}$ in diameter that were observed on CT or MRI, or revealed by FDG-PET were considered to be metastases. The patients were assigned to a clinical stage on the basis of the FIGO classification.

Treatment schedule. CRT consisted of an external beam of radiation therapy (EBRT) to the whole pelvis followed by a high-dose rate of intra-cavitary brachytherapy (HDR-ICBT) and platinum-based chemotherapy (CTx).

EBRT was administered to the whole pelvis with 6 or $10 \mathrm{MV}$ photon-beams. No patients received prophylactic extended-field radiation therapy (RT). The daily dose was
1.8-2.0 Gy, which was administered to the mid-pelvis once a day, 5 days a week. Depending on the tumor size, the whole pelvis was irradiated using the four-field box technique up to a dose of 20-40 Gy, then a boost up to a dose of 50-50.4 Gy was administered to the parametrium, with a $4 \mathrm{~cm}$-wide central block performed using the antero-posterior parallel two-field technique. If necessary, a boost of $10 \mathrm{~Gy}$ in $2 \mathrm{~Gy}$ fractions was administered to the PLN.

Subsequent to the exposure of the central block to EBRT, HDR-ICBT was started, using an Iridium-192 remote afterloading technique, in 6 Gy fractions, once or twice a week, for a minimum of 3-4 fractions (microSelectron HDR-V3; Elekta AB, Stockholm, Sweden). In the majority of patients, a tandem and ovoid applicator (Elekta $\mathrm{AB}$ ) were used in combination for treatment at the primary tumor (point A), while in patients with a lower vaginal extension, a tandem-cylinder (Elekta) was used for treatment at a distance of $5 \mathrm{~mm}$ from the applicator surface. All HDR-ICBTs were devised based on CT using PLATO software version 14.2.6 (Nucletron, Veenendaal, The Netherlands) for each application.

The cumulative doses administered to the tumor from EBRT and HDR-ICBT, were normalized to the biologically equivalent doses in $2 \mathrm{~Gy}$ fractions $\left(\mathrm{GyEQD}_{2}\right)$ based on the linear-quadratic model using an $\alpha / \beta$ ratio of $10 \mathrm{~Gy}$. The median total dose to point A was $70.9 \mathrm{GyEQD}_{2}$ (range, 53.4-85.6).

CTx was administered on the first day of RT. Between 2001 and 2007, cisplatin was administered at a dose of $75 \mathrm{mg} / \mathrm{m}^{2}$ tri-weekly, for 3 cycles. Subsequent to 2007, 


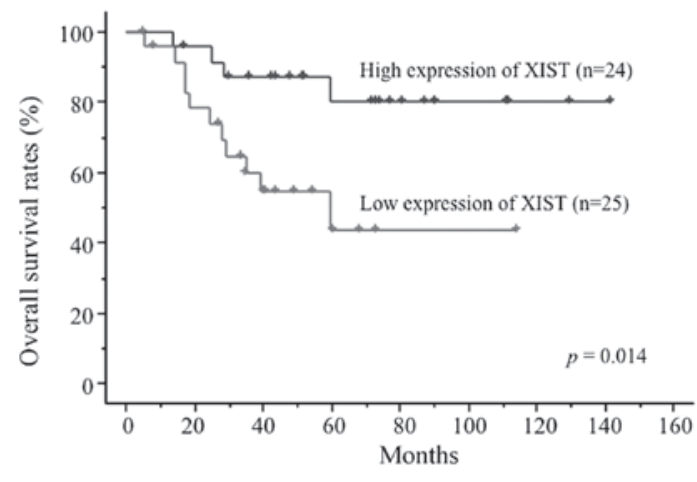

Figure 1. Survival curve demonstrating the overall survival rates for patients with high or low expression of XIST. XIST, X inactive-specific transcript.

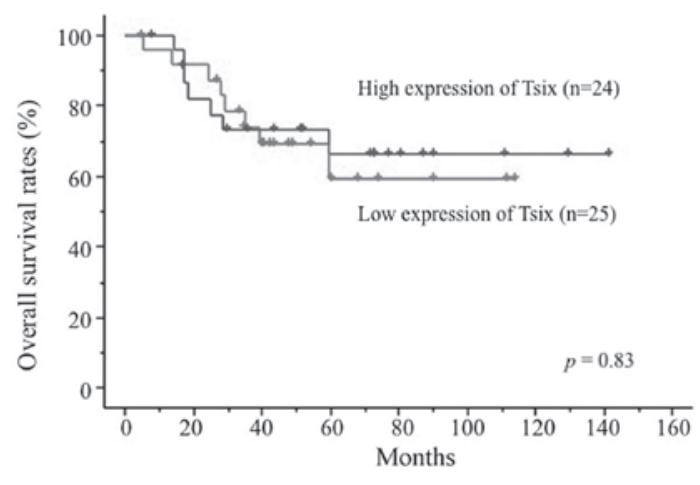

Figure 2. Survival curve demonstrating the overall survival rates for patients with high or low expression of Tsix. cisplatin $\left(40 \mathrm{mg} / \mathrm{m}^{2}\right.$ weekly, for 6 cycles $)$ or nedaplatin (75-100 $\mathrm{mg} / \mathrm{m}^{2}$ tri-weekly, for 3 cycles) became the principal drugs of the CTx regimen. Patients with an advanced age, lower performance status or renal dysfunction required a reduction in the $\mathrm{CTx}$ dose.

Follow-up and analysis of response and survival. Follow-up was performed every month for the first year, every 2-3 months for the second year and 3-6 months thereafter. Follow-up procedures consisted of physical and pelvic examination, cervical Papanicolaou smears and tumor markers. Chest to pelvic CT was performed with an interval of 3-6 months for the first 2 years, and 6-12 months thereafter. Pelvic MRI or FDG-PET was performed if required.

Complete remission was defined as no evidence of disease 3 months subsequent to the end of treatment, as evaluated by clinical and radiographic examinations. A diagnosis of tumor progression or recurrence was based on physical or radiographic examination or pathological confirmation.

Statistical analysis. Statistical analysis was performed using StatView Dataset File software version 5.0J for Windows (SAS Institute, Inc., Cary, NC, USA). The survival period was defined as the time between the start of RT and cancer progression, mortality from any cause or the last follow-up date. Survival curves were estimated using the Kaplan-Meier method, and log-rank tests were used to compare the survival distributions. Differences in patient or tumor characteristics were analyzed by the $\chi^{2}$ test or Fisher's exact test for 2 x 2 columns. Cox proportional hazards model was used for multivariate analysis. $\mathrm{P}<0.05$ was considered to indicate a statistically significant difference.

\section{Results}

The 4-year overall survival (OS) rates were $71.2 \pm 6.8$ for all 49 patients and $73.5 \pm 7.6 \%$ for the 36 patients with FIGO stage III-IVA tumors. Among the 5 lncRNAs, XIST expression was significantly associated with prognosis. The 49 patients were classified into high $(n=24)$ and low $(n=25)$ XIST expression groups, according to the median value of XIST expression. The Kaplan-Meier survival curves demonstrated that XIST expression levels were significantly associated with OS rates. The 4-year OS rates were $87.1 \pm 6.9 \%$ in the high expression group and $54.4 \pm 10.8 \%$ in the low expression group ( $\mathrm{P}=0.014$; Fig. 1). Similarly, the 4-year progression-free survival (PFS) rates were $74.5 \pm 9.0$ and $49.8 \pm 10.3 \%$, in the high and low XIST expression groups, respectively $(\mathrm{P}=0.065)$. By contrast, the expression levels of Tsix, TERC, DHFR upstream transcripts and Air were not associated with prognosis (Fig. 2; Table III). Tumor size was also a variable that was significantly associated with OS rates $(\mathrm{P}=0.035)$. Clinicopathological factors were compared between the high XIST expression and low XIST expression groups (Table IV). XIST upregulation was not associated with any of the clinicopathological factors. Multivariate analysis also demonstrated that the XIST expression level was significantly associated with OS rates (Table V). These results strongly suggest that XIST expression levels may be a potential prognostic factor for cervical cancer OS rates. Therefore, the present study concludes that overexpression of XIST may be important in CSCC progression and development.

\section{Discussion}

The following 5 lncRNAs examined in the present study had been implicated in cancer, according to previous studies (12-14): XIST, Tsix, TERC, DHFR upstream transcripts and Air.

XIST is involved in X chromosome inactivation (XCI) in the cells of females and allows $\mathrm{X}$ chromosome equilibration in males. XIST expression has been downregulated in various human cancers. Loss of XCI and downregulation of XIST expression are commonly observed in basal-like cancer, breast cancer susceptibility gene 1-null triple negative breast cancer (23-29) and ovarian cancer cell lines (30,31). In ovarian cancer cell lines, XIST expression may act as a prognostic marker associated with a chemotherapeutic response (32).

Tsix is transcribed in the antisense orientation from XIST and fully overlaps with the XIST gene (33). Tsix inhibits XIST expression in cis (via interactions between different $\mathrm{X}$ chromosome regions) by several mechanisms. In one mechanism, Tsix binds to complementary XIST RNA and renders it non-functional. Following this binding, XIST is made inactive through dicer, which is a type of endoribonuclease, preferentially cleaves double-stranded RNA (33). However, other mechanisms are also currently being studied. 
Table III. Univariate analysis for OS rate by log-rank test.

\begin{tabular}{|c|c|c|c|c|}
\hline Variable & $\mathrm{n}$ & 2 year OS rate, $\%$ & 4 year OS rate, $\%$ & P-value \\
\hline Age, years & & & & 0.240 \\
\hline$<56$ & 25 & $91.7 \pm 5.6$ & $75.0 \pm 8.8$ & \\
\hline$\geq 56$ & 24 & $82.4 \pm 8.0$ & $67.0 \pm 10.4$ & \\
\hline FIGO stage & & & & 0.780 \\
\hline I-II & 13 & $81.8 \pm 11.6$ & $63.6 \pm 14.5$ & \\
\hline III-IV & 36 & $88.8 \pm 5.3$ & $73.5 \pm 7.6$ & \\
\hline Nodal status & & & & 0.450 \\
\hline No & 32 & $89.9 \pm 5.5$ & $68.0 \pm 8.9$ & \\
\hline N1 & 17 & $82.4 \pm 9.2$ & $76.5 \pm 10.3$ & \\
\hline Max. tumor diameter, $\mathrm{cm}$ & & & & 0.035 \\
\hline$\leq 5$ & 21 & $94.7 \pm 5.1$ & $84.2 \pm 8.4$ & \\
\hline$>5$ & 28 & $82.0 \pm 7.3$ & $61.9 \pm 9.6$ & \\
\hline RT dose, GyEQD 2 & & & & 0.980 \\
\hline$\leq 70$ & 24 & $82.8 \pm 7.8$ & $73.5 \pm 9.3$ & \\
\hline$>70$ & 25 & $91.7 \pm 5.6$ & $68.8 \pm 9.9$ & \\
\hline Initial hemoglobin, $\mathrm{g} / \mathrm{dl}$ & & & & 0.500 \\
\hline$\leq 12$ & 25 & $84.0 \pm 7.3$ & $63.5 \pm 9.7$ & \\
\hline$>12$ & 24 & $90.9 \pm 6.1$ & $80.7 \pm 8.7$ & \\
\hline XIST & & & & 0.014 \\
\hline High & 24 & $95.8 \pm 4.1$ & $87.1 \pm 6.9$ & \\
\hline Low & 25 & $78.4 \pm 8.6$ & $54.4 \pm 10.8$ & \\
\hline Tsix & & & & 0.830 \\
\hline High & 24 & $82.2 \pm 8.1$ & $73.0 \pm 9.4$ & \\
\hline Low & 25 & $91.7 \pm 5.6$ & $69.2 \pm 9.8$ & \\
\hline TERC & & & & 0.910 \\
\hline High & 24 & $87.1 \pm 6.9$ & $74.1 \pm 9.1$ & \\
\hline Low & 25 & $83.3 \pm 7.6$ & $65.5 \pm 10.0$ & \\
\hline DHFR, upstream transcripts & & & & 0.910 \\
\hline High & 24 & $87.1 \pm 6.9$ & $74.1 \pm 9.1$ & \\
\hline Low & 25 & $87.1 \pm 6.9$ & $67.6 \pm 10.2$ & \\
\hline Air & & & & 0.850 \\
\hline High & 24 & $82.9 \pm 7.8$ & $74.2 \pm 9.1$ & \\
\hline Low & 25 & $91.5 \pm 5.8$ & $67.5 \pm 10.3$ & \\
\hline
\end{tabular}

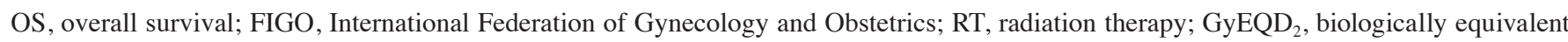
dose in 2 Gy fractions; XIST, X inactive-specific transcript; TERC, telomerase RNA component; DHFR, dihydrofolate reductase; Air, antisense insulin-like growth-factor type-II receptor RNA.

TERC is a component of telomerase that extends telomeres (34). An increase in TERC gene expression has been frequently detected in a variety of human cancers (35). Furthermore, amplification of TERC and overexpression of telomerase are associated with cervical tumorigenesis (36). Therefore, testing whether TERC has been amplified in cervical cancer may be used in addition to cytology screening and human papilloma virus testing, particularly in high-risk patients.

DHFR upstream transcripts are transcribed upstream of the DHFR gene and regulate DHFR expression by forming a triple helix with the promoter and disassociating from the pre-initiation complex (19). It has been reported that this lncRNA may be linked to cancer (13); however, the molecular mechanism remains unidentified. DHFR is the enzyme that converts dihydrofolate into tetrahydrofolate. This reaction is essential for de novo nucleic acid synthesis. DHFR upstream transcripts may affect de novo nucleic acid synthesis and this dysbolism may be conducive to tumorigenesis (37).

The mannose 6-phosphate/insulin-like growth-factor type-II receptor (M6P/IGF-IIR) is considered to act as a suppressor of tumor growth in various types of cancer (38). Air regulates genomic imprinting of a cluster of autosomal genes, including IGF-IIR, Slc22a 2 and Slc22a3 in cis in 
Table IV. Univariate analysis for XIST expression by $\chi^{2}$ test.

\begin{tabular}{|c|c|c|c|}
\hline Variables & $\begin{array}{c}\text { High XIST expression } \\
\text { group, n }(\%)\end{array}$ & $\begin{array}{c}\text { Low XIST expression } \\
\text { group, n }(\%)\end{array}$ & P-value \\
\hline Total & $24(100)$ & $25(100)$ & \\
\hline \multicolumn{4}{|l|}{ Age, years } \\
\hline$\leq 55$ & $15(63)$ & $10(40)$ & \multirow[t]{2}{*}{0.12} \\
\hline$\geq 56$ & $9(38)$ & $15(60)$ & \\
\hline \multicolumn{4}{|c|}{ FIGO stage } \\
\hline I-II & $6(25)$ & $7(28)$ & \multirow[t]{2}{*}{0.81} \\
\hline III-IV & $18(75)$ & $18(72)$ & \\
\hline \multicolumn{4}{|c|}{ Nodal status } \\
\hline No & $13(54)$ & $19(76)$ & \multirow[t]{2}{*}{0.11} \\
\hline N1 & $11(46)$ & $6(24)$ & \\
\hline \multicolumn{4}{|c|}{ Maximum tumor diameter, $\mathrm{cm}$} \\
\hline$\leq 5$ & $10(42)$ & $11(44)$ & \multirow[t]{2}{*}{0.87} \\
\hline$>5$ & $14(58)$ & $14(56)$ & \\
\hline \multicolumn{4}{|c|}{ RT dose, GyEQD 2} \\
\hline$\leq 70$ & $14(58)$ & $10(40)$ & \multirow[t]{2}{*}{0.20} \\
\hline$>70$ & $10(42)$ & $15(60)$ & \\
\hline \multicolumn{4}{|c|}{ Initial hemoglobin, $\mathrm{g} / \mathrm{dl}$} \\
\hline$\leq 12$ & $12(50)$ & $13(52)$ & \multirow[t]{2}{*}{0.89} \\
\hline$>12$ & $12(50)$ & $12(48)$ & \\
\hline
\end{tabular}

XIST, X inactive-specific transcript; FIGO, International Federation of Gynecology and Obstetrics; RT, radiation therapy; GyEQD ${ }_{2}$, biologically equivalent dose in 2 Gy fractions.

mouse chromosome 17 (39). Although full-length transcripts have yet to be characterized in humans, this lncRNA may be associated with human cancers (13) and may affect cancer-associated gene expression at an epigenetic level (40).

To the best of our knowledge, the present study is the first to demonstrate an association between the XIST expression level and the prognosis of CSCC treated with platinum-based CRT. High expression levels of XIST were clinically associated with increased OS rates. This observation reinforces the theory that XIST lncRNA may be used to predict the prognosis of CSCC treated with CRT.

Since lncRNAs are produced from the majority of the regions in the genome, they are emerging as key molecules in human cancer and may be useful as novel biomarkers for the diagnosis, prognosis and prediction of response to treatment. In particular, IncRNAs have been hypothesized to possess tumor suppressive and oncogenic functions in various cancer types. Homeobox transcript antisense intergenic RNA (HOTAIR) has been reported to be associated with the progression and prognosis of cancers, including breast, esophageal, lung, liver and endometrial carcinomas (41-46). In endometrial carcinoma, an increased level of HOTAIR was demonstrated to be associated with the depth of myometrial invasion, lymphovascular space invasion and a poorer OS rate. Therefore, this lncRNA may be a novel biomarker of prognosis in cancer patients (47).

The XIST gene is located in the X chromosome inactivation center and its product is transcribed from the inactive
X chromosome $(48,49)$. XIST then spreads along the $X$ chromosome from which it was transcribed. XIST IncRNA is important in the initiation of XCI in female cells, which achieves dosage equilibration of X-linked genes with males. Since XCI silences several hundred genes, including oncogenes, misexpression of XIST may potentially be a mechanism underlying tumorigenesis (49-51). Recently, Yildirim et al demonstrated a causal link between XIST expression and cancer in mice (52). Deleting XIST in the mouse blood compartment was found to lead to a highly aggressive myeloproliferative neoplasm and myelodysplastic syndrome with $100 \%$ penetrance (52). This result suggests that upregulation of X-linked genes following the deletion of XIST leads to genome-wide alterations in key developmental and homeostatic pathways, which in turn drive cancer formation and progression (52). Furthermore, in theory, XIST is particularly important in female-specific cancer. Certain studies have claimed that XIST is involved in female cancers (14,23-32). Taken together, these findings, and the results from the present study, demonstrate that a low XIST level may lead to the depression of $\mathrm{X}$ chromosome inactivation and the continuous gain of $\mathrm{X}$ chromosome reactivation. This phenomenon may, in turn, upregulate the expression of numerous cancer-associated genes, including genes responsible for the aggressiveness of cancer, in the X chromosome. This may lead to low XIST group patients not being treated effectively with CRT. In addition, high XIST levels may 
Table V. Multivariate analysis for XIST expression OS rates by Cox proportional hazards model.

\begin{tabular}{lccc}
\hline Variables & HR & 95\% CI & P-value \\
\hline Age & & & 0.027 \\
$\quad$ XIST, high vs. low & 0.27 & $0.08-0.86$ & 0.330 \\
Age, $<56$ vs. $\geq 56$, years & 0.59 & $0.21-1.70$ & 0.023 \\
FIGO stage & & & 1.000 \\
$\quad$ XIST, high vs. low & 0.26 & $0.08-0.83$ & 0.028 \\
$\quad$ FIGO stage, I-II vs. III-IV & 1.00 & $0.31-3.22$ & 0.800 \\
$\quad$ Nodal status & 0.27 & $0.08-0.87$ & 0.012 \\
$\quad$ XIST, high vs. low & 1.16 & $0.36-3.73$ & 0.027 \\
$\quad$ Nodal status, N0 vs. N1 & & & $0.06-0.71$ \\
$\quad$ Max. tumor diameter & 0.21 & $0.06-0.84$ & \\
$\quad$ XIST, high vs. low & 0.23 & & \\
Max. tumor diameter, $\leq 5$ vs. $>5, \mathrm{~cm}$ & &
\end{tabular}

XIST, X inactive-specific transcript; OS, overall survival; HR, hazard ratio; CI, confidence interval; FIGO, International Federation of Gynecology and Obstetrics.

trigger the reduction of numerous genes responsible for RT and/or CTx resistance in the $\mathrm{X}$ chromosome, and this may boost the effectiveness of RT and CTx.

Since XIST is able to control cancer, it may be reasonable to propose the reactivation of XIST as a therapeutic strategy in cancer. In tumors, XIST expression may be reactivated by small molecules, such as XIST expression vector, offering a novel therapeutic approach that would target epigenetically functional lncRNAs. If molecules that induce XIST expression are identified, artificially improving the prognosis of a patient may become feasible.

It is notable that there was no association between XIST and Tsix in the present study. In general, Tsix is transcribed from the antisense IncRNA XIST and controls XIST upregulation, and XIST and Tsix often work in combination (53). However, the inherent functions of XIST are considered to be associated with the prognosis of CSCC. The findings of the present study may lead to an improved understanding of the emerging roles of XIST in cancer therapy.

Comprehensive analysis of gene expression using RNA from fresh frozen tumor specimens is important to improve the understanding of cancer pathogenesis, progression and prognosis. However, it is challenging for studies to treat frozen tumor specimens with degradable biomolecules, as frozen tissue samples are not readily available. By contrast, since all biomolecules are fixed, FFPE tissues may be treated at room temperature for extended periods of time, and therefore FFPE tissues are the most widely available specimens. Although formalin may cause the degradation and modification of nucleic acids, leading to a poor recovery of nucleic acid from preserved tissues, presently there are various commercially available kits for the isolation of nucleic acids from FFPE tissue sections (15-18).

Various types of protein markers for the prognosis of cancer have been explored by numerous studies. At present, IncRNAs, in addition to HOTAIR and XIST, may also be considered as candidates for prognostic biomarker roles. Experiments to investigate the role lncRNAs play in cancer may be performed using FFPE specimens routinely preserved in numerous hospitals or laboratories. Additionally, as RT-qPCR possesses a high quantitative capability, precisely-analyzed data may be obtained. In the present study, only $5 \operatorname{lncRNAs}$ were analyzed; there may be other lncRNAs that are associated with the prognosis of CSCC patients. Additional studies are required to investigate other lncRNAs that may associate with cancer prognosis and the mechanisms by which they affect cancer.

There were certain limitations to the present study. First, due to the nature of a retrospective study, there was a lack of consistency of treatment. Second, the present study investigated a small sample size. Over a 13 -year period, 263 patients were referred for definitive RT for cervical cancer treatment at the University of Tokyo Hospital. Of these 263 patients, only 49 were included in the present study. The following patients were excluded from the present study: 104 patients that partially received HDR-ICBT at the University of Tokyo Hospital and received EBRT and CTx at other institutions; 4 patients with other malignancies; 8 patients that received prior treatment for cervical cancer; 7 patients without detailed information of treatment or without follow-up; 14 patients with carcinoma in situ or FIGO stage IA, IB1 or IIA1 without PLN metastasis; 18 patients with para-aortic lymph node metastasis; 3 patients with other distant metastasis; 3 patients with distant metastasis and para-aortic lymph node metastasis; and 1 patient that did not complete the planned RT. Out of the remaining 101 patients, 76 patients received CRT and 25 received RT alone. Among the 76 patients treated with CRT, 58 were diagnosed with squamous cell carcinoma. The FFPE tissue samples from 9 patients were unsuitable or lost. Therefore, the remaining 49 patients were included in the present study. To minimize variation that may have affected prognosis, the eligibility criteria were specific, and so the data gathered in the present study were reliable. 
In conclusion, the results of the present study indicate that the expression of XIST associates with the OS rates of CSCC patients. However, additional studies are required to investigate the underlying mechanisms of XIST associated with prognosis, including the regulation of the response to CRT or progression of CSCC. Additional studies may reveal novel therapeutic strategies for CSCC treatment.

\section{References}

1. NCI Press Office: NCI issues clinical announcement on cervical cancer: Chemotherapy plus radiation improves survival. http://www.nih.gov/news/pr/feb99/nci-22.htm. Accessed August 28, 2014

2. NCCN Guidelines ${ }^{\circledR}$ : NCCN clinical practice guidelines in oncology: Cervical cancer version 1,2016. National Comprehensive Cancer Network, Inc., Fort Washington, PA, USA, 2015.

3. Chemoradiotherapy for Cervical Cancer Meta-Analysis Collaboration: Reducing uncertainties about the effects of chemoradiotherapy for cervical cancer: A systematic review and meta-analysis of individual patient data from 18 randomized trials. J Clin Oncol 26: 5802-5812, 2008.

4. Nakano T, Ohno T, Ishikawa H, Suzuki Y and Takahashi T: Current advancement in radiation therapy for uterine cervical cancer. J Radiat Res 51: 1-8, 2010.

5. Hauspy J, Harley I and Fyles AW: Uterine cervix cancer. In: Prognostic Factors in Cancer. Gospodarowicz MK, O'Sullivan B and Sobin LH (eds). 3rd edition. John Wiley \& Sons Inc., Hoboken, NJ, pp219-222, 2006.

6. Noordhuis MG, Eijsink JJ, Roossink F, de Graeff P, Pras E, Schuuring E, Wisman GB, de Bock GH and van der Zee AG: Prognostic cell biological markers in cervical cancer patients primarily treated with (chemo)radiation: A systematic review. Int J Radiat Oncol Biol Phys 79: 325-334, 2011.

7. Harima Y, Ikeda K, Utsunomiya K, Komemushi A, Kanno S, Shiga $\mathrm{T}$ and Tanigawa N: Apolipoprotein C-II is a potentia serum biomarker as a prognostic factor of locally advanced cervical cancer after chemoradiation therapy. Int J Radiat Oncol Biol Phys 87: 1155-1161, 2013.

8. Cech TR and Steitz JA: The noncoding RNA revolution-trashing old rules to forge new ones. Cell 157: 77-94, 2014.

9. Elgar G and Vavouri T: Tuning in to the signals: Noncoding sequence conservation in vertebrate genomes. Trends Genet 24: 344-352, 2008

10. Zhang Y, Yang L and Chen LL: Life without A tail: New formats of long noncoding RNAs. Int J Biochem Cell Biol 54: 338-349, 2014.

11. Hu X, Schwarz JK, Lewis JS Jr, Huettner PC, Rader JS, Deasy JO, Grigsby PW and Wang XA: A microRNA expression signature for cervical cancer prognosis. Cancer Res 70: 1441-1448, 2010.

12. Hauptman $\mathrm{N}$ and Glavač D: Long non-coding RNA in cancer. Int J Mol Sci 14: 4655-4669, 2013.

13. Spizzo R, Almeida MI, Colombatti A and Calin GA: Long non-coding RNAs and cancer: A new frontier of translational research? Oncogene 31: 4577-4587, 2012.

14. Cheetham SW, Gruhl F, Mattick JS and Dinger ME: Long noncoding RNAs and the genetics of cancer. Br J Cancer 108 2419-2425, 2013.

15. Ismailov S, Rashitov M, Kobayashi M, Shibata N, Kato Y, Omi Y, Iihara M and Okamoto T: Trefoil factor 3 (TFF3) mRNA expression level in follicular thyroid tumors using formalin-fixed, paraffin-embedded (FFPE) blocks. O J Pathol 3: 78-84, 2013

16. von Ahlfen S, Missel A, Bendrat K and Schlumpberger M: Determinants of RNA quality from FFPE samples. PLoS One. 2 e1261, 2007.

17. Sánchez-Navarro I, Gámez-Pozo A, González-Barón M, Pinto-Marín A, Hardisson D, López R, Madero R, Cejas P, Mendiola M, Espinosa E and Vara JA: Comparison of gene expression profiling by reverse transcription quantitative PCR between fresh frozen and formalin-fixed, paraffin-embedded breast cancer tissues. Biotechniques 48: 389-397, 2010.

18. Mittempergher L, de Ronde JJ, Nieuwland M, Kerkhoven RM, Kerkhoven RM, Simon I, Rutgers EJ, Wessels LF and Van't Veer LJ: Gene expression profiles from formalin fixed paraffin embedded breast cancer tissue are largely comparable to fresh frozen matched tissue. PLoS One. 6: e17163, 2011
19. Martianov I, Ramadass A, Serra Barros A, Chow N and Akoulitchev A: Repression of the human dihydrofolate reductase gene by a non-coding interfering transcript. Nature 445: 666-670, 2007.

20. Miyagawa R, Tano K, Mizuno R, Nakamura Y, Ijiri K, Rakwal R, Shibato J, Masuo Y, Mayeda A, Hirose T and Akimitsu N: Identification of cis- and trans-acting factors involved in the localization of MALAT-1 noncoding RNA to nuclear speckles. RNA 18: 738-751, 2012

21. Kumamoto T and Oshio S: Effect of fetal exposure to bisphenol A on brain mediated by X-chromosome inactivation. J Toxicol Sci 38: 485-494, 2013.

22. Latos PA, Pauler FM, Koerner MV, et al: Airn transcriptional overlap, but not its lncRNA products, induces imprinted Igf2r silencing. Science 338: 1469-1472, 2012.

23. Ganesan S, Silver DP, Greenberg RA, Avni D, Drapkin R, Miron A, Mok SC, Randrianarison V, Brodie S, Salstrom J, et al: BRCA1 supports XIST RNA concentration on the inactive X chromosome. Cell 111: 393-405, 2002.

24. Pageau GJ, Hall LL and Lawrence JB: BRCA1 does not paint the inactive $\mathrm{X}$ to localize XIST RNA but may contribute to broad changes in cancer that impact XIST and Xi heterochromatin. J Cell Biochem 100: 835-850, 2007.

25. Richardson AL, Wang ZC, De Nicolo A, Lu X, Brown M, Miron A, Liao X, Iglehart JD, Livingston DM and Ganesan S: $\mathrm{X}$ chromosomal abnormalities in basal-like human breast cancer. Cancer Cell 9: 121-132, 2006.

26. Silver DP, Dimitrov SD, Feunteun J, Gelman R, Drapkin R, Lu SD, Shestakova E, Velmurugan S, Denunzio N, Dragomir S, et al: Further evidence for BRCA1 communication with the inactive $\mathrm{X}$ chromosome. Cell 128: 991-1002, 2007.

27. Sirchia SM, Ramoscelli L, Grati FR, Barbera F, Coradini D, Rossella F, Porta G, Lesma E, Ruggeri A, Radice P, et al: Loss of the inactive $\mathrm{X}$ chromosome and replication of the active $\mathrm{X}$ in BRCA1-defective and wild-type breast cancer cells. Cancer Res 65: 2139-2146, 2005.

28. Sirchia SM, Tabano S, Monti L, Recalcati MP, Gariboldi M, Grati FR, Porta G, Finelli P, Radice P and Miozzo M: Misbehaviour of XIST RNA in breast cancer cells. PLoS One 4: e5559, 2009.

29. Vincent-Salomon A, Ganem-Elbaz C, Manié E, Raynal V, Sastre-Garau X, Stoppa-Lyonnet D, Stern MH and Heard E: $X$ inactive-specific transcript RNA coating and genetic instability of the X chromosome in BRCA1 breast tumors. Cancer Res 67: 5134-5140, 2007.

30. Benoît MH, Hudson TJ, Maire G, Squire JA, Arcand SL, Provencher D, Mes-Masson AM and Tonin PN: Global analysis of chromosome $\mathrm{X}$ gene expression in primary cultures of normal ovarian surface epithelial cells and epithelial ovarian cancer cell lines. Int J Oncol 30: 5-17, 2007.

31. Kawakami T, Zhang C, Taniguchi T, Kim CJ, Okada Y, Sugihara H, Hattori T, Reeve AE, Ogawa O and Okamoto K: Characterization of loss-of-inactive $\mathrm{X}$ in Klinefelter syndrome and female-derived cancer cells. Oncogene 23: 6163-6169, 2004.

32. Huang KC, Rao PH, Lau CC, Heard E, Ng SK, Brown C, Mok SC, Berkowitz RS and Ng SW: Relationship of XIST expression and responses of ovarian cancer to chemotherapy. Mol Cancer Ther 1: 769-776, 2002.

33. Lee JT, Davidow LS and Warshawsky D: Tsix, a gene antisense to Xist at the X-inactivation centre. Nat Genet 21:400-404, 1999.

34. Feng J, Funk WD, Wang SS, Weinrich SL, Avilion AA, Chiu CP, Adams RR, Chang E, Allsopp RC, Yu J, et al: The RNA component of human telomerase. Science 269: 1236-1241, 1995.

35. Cao Y, Bryan TM and Reddel RR: Increased copy number of the TERT and TERC telomerase subunit genes in cancer cells. Cancer Sci 99: 1092-1099, 2008.

36. Tu Z, Zhang A, Wu R, Jiang J, Li Y, Wulan N, Li J, Zhang Y, Li Y, Chen Z and Wei L: Genomic amplification of the human telomerase RNA gene for differential diagnosis of cervical disorders. Cancer Genet Cytogenet 191: 10-16, 2009.

37. Hitchings GH Jr: Nobel lecture in physiology or medicine - 1988 Selective inhibitors of dihydrofolate reductase. In Vitro Cell Dev Biol 25: 303-310, 1989.

38. El-Shewy HM and Luttrell LM: Insulin-like growth factor-2/mannose-6 phosphate receptors. Vitam Horm 80: 667-697, 2009

39. Nagano T, Mitchell JA, Sanz LA, Pauler FM, Ferguson-Smith AC, Feil R and Fraser P: The Air noncoding RNA epigenetically silences transcription by targeting G9a to chromatin. Science 322: 1717-1720, 2008

40. Mohammad F1, Mondal T and Kanduri C: Epigenetics of imprinted long noncoding RNAs. Epigenetics 4: 277-286, 2009. 
41. Gupta RA, Shah N, Wang KC, Kim J, Horlings HM, Wong DJ, Tsai MC, Hung T, Argani P, Rinn JL, et al: Long non-coding RNA HOTAIR reprograms chromatin state to promote cancer metastasis. Nature 464: 1071-1076, 2010.

42. Chisholm KM, Wan Y, Li R, Montgomery KD, Chang HY and West RB: Detection of long non-coding RNA in archival tissue: Correlation with polycomb protein expression in primary and metastatic breast carcinoma. PLoS One 7: e47998, 2012.

43. Chen FJ, Sun M, Li SQ, Wu QQ, Ji L, Liu ZL, Zhou GZ, Cao G, Jin L, Xie HW, et al: Upregulation of the long non-coding RNA HOTAIR promotes esophageal squamous cell carcinoma metastasis and poor prognosis. Mol Carcinog 52: 908-915, 2013.

44. Liu XH, Liu ZL, Sun M, Liu J, Wang ZX and De W: The long non-coding RNA HOTAIR indicates a poor prognosis and promotes metastasis in non-small cell lung cancer. BMC Cancer 13: 464, 2013.

45. Yang Z, Zhou L, Wu LM, Lai MC, Xie HY, Zhang F and Zheng SS: Overexpression of long non-coding RNA HOTAIR predicts tumor recurrence in hepatocellular carcinoma patients following liver transplantation. Ann Surg Oncol 18: 1243-1250, 2011.

46. Geng YJ, Xie SL, Li Q, Ma J and Wang GY: Large intervening non-coding RNA HOTAIR is associated with hepatocellular carcinoma progression. J Int Med Res 39: 2119-2128, 2011.
47. He X, Bao W, Li X, Chen Z, Che Q, Wang H and Wan XP: The long non-coding RNA HOTAIR is upregulated in endometrial carcinoma and correlates with poor prognosis. Int J Mol Med 33: 325-332, 2014

48. Brown CJ, Ballabio A, Rupert JL, Lafreniere RG, Grompe M, Tonlorenzi R and Willard HF: A gene from the region of the human $\mathrm{X}$ inactivation centre is expressed exclusively from the inactive X chromosome. Nature 349: 38-44, 1991.

49. Weakley SM, Wang H, Yao Q and Chen C: Expression and function of a large non-coding RNA gene XIST in human cancer. World J Surg 35: 1751-1756, 2011.

50. Liu Y, Wang L and Zheng P: X-linked tumor suppressors: Perplexing inheritance, a unique therapeutic opportunity. Trends Genet 26: 260-265, 2010.

51. Spatz A, Borg C and Feunteun J: X-chromosome genetics and human cancer. Nat Rev Cancer 4: 617-629, 2004.

52. Yildirim E, Kirby JE, Brown DE, Mercier FE, Sadreyev RI, Scadden DT and Lee JT: Xist RNA is a potent suppressor of hematologic cancer in mice. Cell 152: 727-742, 2013.

53. Froberg JE, Yang L and Lee JT: Guided by RNAs: X-inactivation as a model for lncRNA function. J Mol Biol 425: 3698-3706, 2013. 